\title{
A complex valve anatomy for Mitraclip: Forme fruste of Barlow's disease with cleft
}

\author{
Giuliana Capretti, Eustachio Agricola, Matteo Montorfano, Alaide Chieffo \\ Interventional Cardiology Unit, Cardio-Thoracic-Vascular Department, \\ San Raffaele Scientific Institute, Milan, Italy
}

\begin{abstract}
A 84-year-old man was admitted to our hospital for severe symptomatic mitral regurgitation (MR). The echocardiography showed a degenerative forme fruste of Barlow's disease with a prolapse of P2-M2 scallop. Additionally, a P2-M1 flail caused by ruptured chordae, and an isolated cleft between P1-P2 (Fig. 1A, Suppl. Video 1) were also identified. Despite the challenging anatomy, Heart Team reached consensus for MitraClip (Abbott Vascular) implantation because of high operative risk and frailty.

Initially, one single clip was placed in a central position between A2-P2 scallops resulting in a moderate $\mathrm{MR}$ with no significant residual stenosis (Fig. 1B). Despite the presence of the cleft, a second clip was implanted in a lateral position, parallel to the first clip (Fig. 1C). The procedure resulted
\end{abstract}

in mild MR with a double orifice valve and mean gradient of $3 \mathrm{mmHg}$ (Fig. 1D, E, Suppl. Video 2).

Complex Barlow's disease represents an unfavourable anatomical condition for MitraClip. In this case, the coexistence of a flail and a cleft represented an additional challenge. The presence of a cleft is usually regarded as an inappropriate condition for MitraClip since the cleft can limit the areas of possible grasping and the clip could worsen MR by opening the cleft.

Few cases of convergent clips implantation have been described in this lesion subset. According to the literature, this is the first reported case of parallel clips implantation which highlights that MitraClip procedure performed by experienced hands could lead to good results even in those patients who present an adverse or challenging anatomy.

\section{Conflict of interest: None declared}

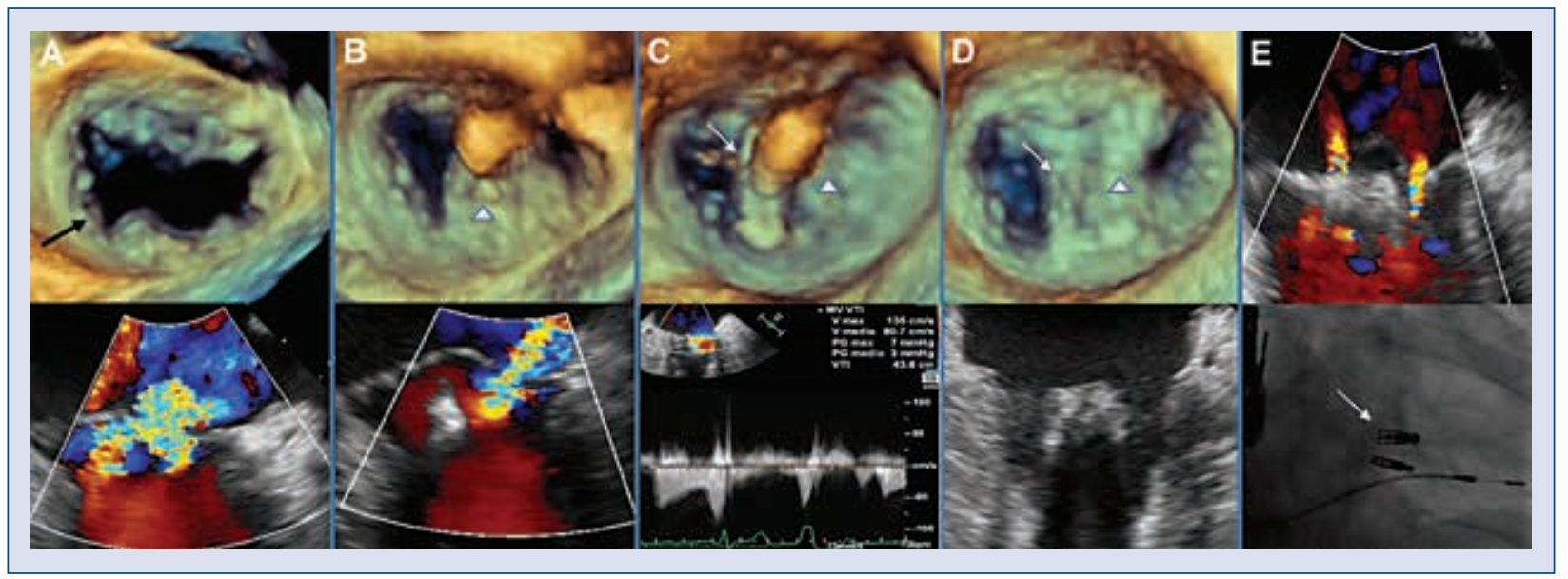

Figure 1. A. Preprocedural three-dimensional (3D) transesophageal echocardiography (TEE) atrial view of the mitral valve. Black arrow indicates the cleft; B. Intraprocedural 3D TEE after implantation of the first clip (arrowhead); C. Intraprocedural 3D TEE during implantation of the second clip (arrow) and final mean trans-valve gradient; D. Postprocedural 3D and 2D TEE showing the two clips; E. Postprocedural TEE Doppler showing mild mitral regurgitation and fluoroscopic image of the two clips (arrow: second clip in lateral position).

Address for correspondence: Giuliana Capretti, MD, Interventional Cardiology Unit, San Raffaele Scientific Institute, Via Olgettina 6020132 Milan, Italy, tel: +390226437362, fax: +390226437339, e-mail: giuliana.capretti@gmail.com 